\title{
Strange Particle Reconstruction by the Missing Mass Method
}

\author{
Pavel Kisel ${ }^{1,3,4, \star}$, Ivan Kisel ${ }^{1,2,3}$, Peter Senger ${ }^{3}$, louri Vassiliev ${ }^{3}$, and Maksym Zyzak ${ }^{3}$ \\ ${ }^{1}$ Goethe University Frankfurt, Frankfurt am Main, Germany \\ ${ }^{2}$ FIAS Frankfurt Institute for Advanced Studies, Frankfurt am Main, Germany \\ ${ }^{3}$ GSI Helmholtz Center for Heavy Ion Research, Darmstadt, Germany \\ ${ }^{4}$ JINR Joint Institute for Nuclear Research, Dubna, Russia
}

\begin{abstract}
The main goal of modern heavy-ion experiments is a comprehensive study of the QCD phase diagram, in a region of Quark-Gluon Plasma (QGP) and possible phase transition to QGP phase.

Strange particles produced in the collision are sensitive probes of the created media. Reconstruction of $\Sigma$ particles together with other strange particles completes the picture of strangeness production. $\Sigma^{+}$and $\Sigma^{-}$have all decay modes with at least one neutral daughter, which can not be registered by the CBM detector.

For their identification the missing mass method is proposed: a) tracks of the mother $\left(\Sigma^{-}\right)$ and the charged daughter $\left(\pi^{-}\right)$particles are reconstructed in the tracking system; b) the neutral daughter particle $(n)$ is reconstructed from these tracks; c) a mass constraint is set on the reconstructed neutral daughter; $d$ ) the mother particle is constructed of the charged and reconstructed neutral daughter particles and the mass spectrum is obtained, by which the particle can be identified.

The method can be applied for other strange particles too. In total 18 particle decays with neutral daughter are now included into physics analysis.
\end{abstract}

\section{Introduction}

The Compressed Baryonic Matter (CBM) experiment is future fixed-target heavy ion experiment, which will focus on a comprehensive study of the phase diagram of strongly interacting matter and a study of the equation of state of the matter at extremely high baryonic densities [1]. Strangeness production is a sensitive probe of the media created in the collision. Being abundant particles, $\Sigma^{+}$ and $\Sigma^{-}$carry out large fraction of produced strange quarks. Reconstruction of $\Sigma$ together with other strange particles completes the picture of strangeness production and allows to compare yields of $\Sigma$ and $\Sigma^{*}$. Reconstruction of $\Sigma$-particles will open a possibility to investigate $H$-dybarion objects, if such exist, by the decay channel $\Sigma^{-} p$, which is expected to be the dominant one.

All strange mesons and hyperons, except $\Sigma$-particles, can be either registered directly by the Micro Vertex Detector (MVD) and the Silicon Tracking System (STS) of the CBM experiment and identified by the particle identification (PID) detectors, including Ring Image Cherenkov, Transition Radiation, Muon Chambers, and Time of Flight (ToF) detectors, or have decay modes with all charged particles and, as a result, can be reconstructed through their daughter particles. $\Sigma^{+}$and $\Sigma^{-}$have all decay

\footnotetext{
^e-mail: P.Kisel@gsi.de
} 
modes with at least one neutral daughter, which can not be registered by the CBM detector at all (decay modes with $n$ or $v$ ) or can be registered with an efficiency of the $10^{-7}$ level (decay modes with $\left.\pi^{0}\right)$.

The tracking detectors of the CBM experiment will be positioned close enough to the collision point to detect $\Sigma$-hyperons: $c \tau_{\Sigma^{+}}=2.4 \mathrm{~cm}$ and $c \tau_{\Sigma^{-}}=4.4 \mathrm{~cm}$, the first station of MVD will be placed at about $5 \mathrm{~cm}$ and the first station of the STS detector - at $30 \mathrm{~cm}$ from the target position downstream the beam. Thus, the CBM experiment provides perfect capabilities for registration of the $\Sigma$-hyperons, however, these particles can not reach the ToF detector to be identified. The missing mass method is proposed to be applied for their identification, that expands the physics potential of the CBM experiment.

\section{Implementation of the missing mass method}

The missing mass method is based on the conservation laws of the energy and momentum.

$$
\begin{aligned}
M_{\text {miss }}^{2} & =\left(P_{\text {mother }}-P_{\text {ch. daughter }}\right)^{2}, \\
P & =\left(E, p_{x}, p_{y}, p_{z}\right) .
\end{aligned}
$$

the $P$ stay for 4-momentum vectors. Using these formulas the 4-vector of the missing particle can be reconstructed, thus, its mass can be determined.

A typical instance is the decay $\Sigma^{-} \rightarrow n \pi^{-}$. The lifetime $c \tau_{\Sigma^{-}}=4.4 \mathrm{~cm}$, but with the relativistic boost the average length of flight can reach up to $15-20 \mathrm{~cm}$. This is enough for $\Sigma^{-}$to cross $3-$ 4 detector layers producing measurements there. Thus, the $\Sigma^{-}$hyperon can leave the track in the detector system, that can be reconstructed using the Cellular Automaton [2] and the Kalman Filter [3], the main algorithms of the First Level Event Selection (FLES) software package [4]. Since the decay take place in this case insight the detector system, a pion produced in the decay can can also cross several layers and can be detected. Moreover, it can reach the ToF detector and be identified.

The invariant mass of the neutron can be found from two reconstructed $\Sigma^{-}$and $\pi^{-}$particles:

$$
M_{n}^{2}=\left(P_{\Sigma}-P_{\pi}\right)^{2} .
$$

The track of $\Sigma^{-}$hyperon can not be identified directly. Therefore the table mass of the $\Sigma^{-}$hyperon is assumed for all particles, which are considered as candidates to be $\Sigma^{-}$. Under such an assumption a 4-momentum vector is constructed. Currently, only primary particles are considered as $\Sigma^{-}$candidates, that are produced directly in the collision or as a result of the resonance decays. Two cases can occur (see Fig. 1):

- if it was a real $\Sigma^{-}$, then the reconstructed particle candidate will result a mass, which will form a peak in the region of the neutron mass $0.939 \mathrm{GeV} / \mathrm{c}^{2}$;

- otherwise, if the hypothesis is wrong, it will form a continuous combinatorial background and physics background created by other particle decays, for example, $\Xi^{-} \rightarrow \Lambda \pi^{-}$.

Then the mass constraint is set to the neutron candidate using the table mass of the neutron. In such a way, the whole 4-momentum vector of the candidate is modified, that improves the momentum resolution for true particles. In order to get a fully constructed $\Sigma^{-}$candidate, the obtained neutron candidate is added with the $\pi^{-}$daughter particle candidate. As a result, a fully fitted $\Sigma^{-}$candidate is constructed. In such a way, the reconstructed $\Sigma^{-}$particles contain information not only from the track they leaved in the detector, but from the daughter $\pi^{-}$track as well through the reconstructed decay vertex, that allows to improve the resolution of $\Sigma^{-}$momentum. Like in case of the neutron candidates, 

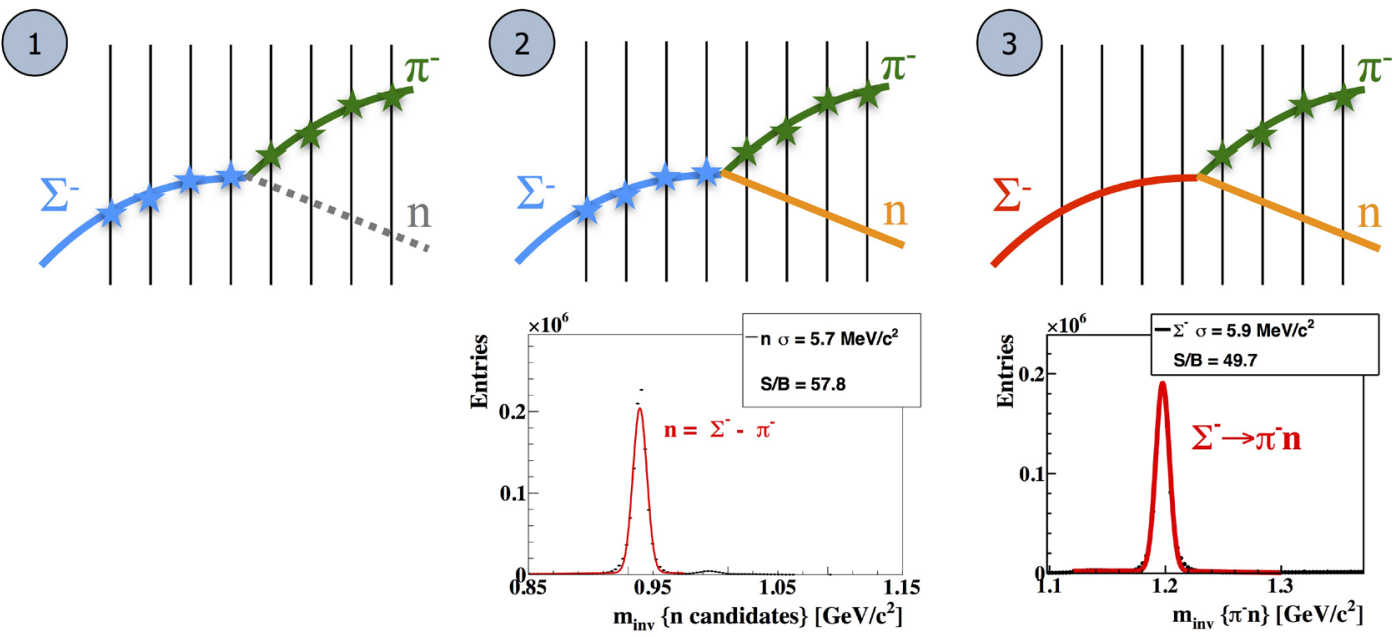

Figure 1. Three stages of the missing mass method implementation in the CBM experiment with illustration of the mass spectra of the reconstructed neutron and $\Sigma^{-}$candidates obtained in 5 millions simulated AuAu central UrQMD events at $10 \mathrm{AGeV}$.

true particles form a peak in the region of $1.197 \mathrm{GeV} / \mathrm{c}^{2}$, that corresponds to $\Sigma^{-}$, while incorrect candidates form a continuous background. And $\Sigma^{-}$particles are identified by the reconstructed mass.

Thus, the algorithm for reconstruction of $\Sigma$-hyperons by the missing mass method consists of three stages (Fig. 1):

1. find tracks of $\Sigma^{-}$and its charged daughter in the tracking system;

2. reconstruct a neutral daughter from the mother and the charged daughter;

3. reconstruct $\Sigma^{-}$mass spectrum from the charged and obtained neutral daughters.

The method has been added to the KF Particle Finder package [5] for short-lived particles reconstruction, that is based on the Kalman filter mathematics.
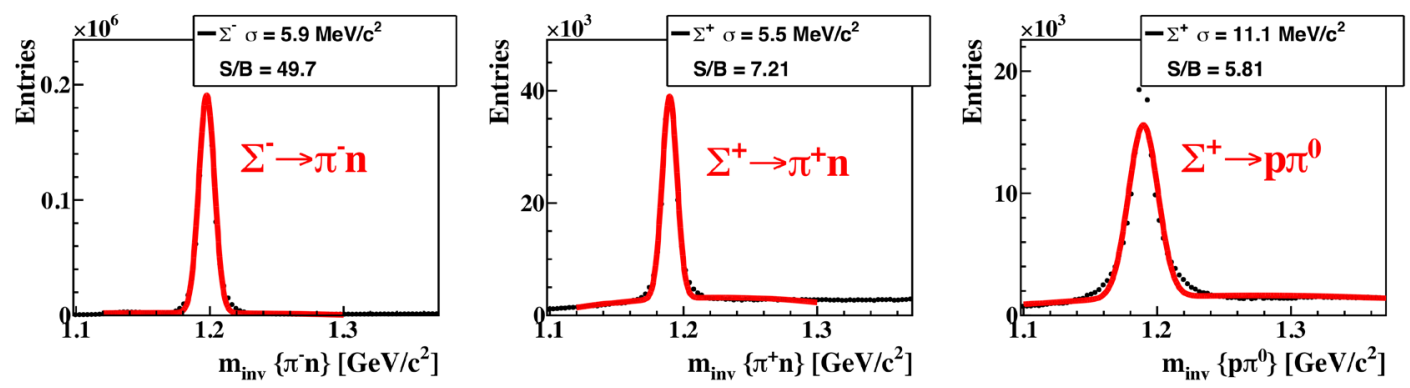

Figure 2. Mass distributions of reconstructed $\Sigma^{-} \rightarrow n \pi^{-}, \Sigma^{+} \rightarrow n \pi^{+}$and $\Sigma^{+} \rightarrow p \pi^{0}$ in 5 millions simulated AuAu central UrQMD events at $10 \mathrm{AGeV}$. 
The topology of decays allows to require the decay point to be positioned after the last hit of a mother track and before the first hit of a daughter track. A cut on $z$ position of the reconstructed decay point reduces a significant part of the combinatorial background.

The comprehensive study of the $\Sigma$ decays has shown that a large fraction of the residual background comes from misinterpretation of the tracks, which were reconstructed in two parts (clones). Since the reconstructed momentum of both parts is the same, the resulting values of rapidity and momentum for a neutral daughter candidate are close to 0 and are well separated from the signal region. Thus, such fake candidates can be strongly suppressed by cutting on the low momentum of the neutral candidate.

The method has been developed and tested with 5 millions simulated AuAu central UrQMD $[6,7]$ events at $10 \mathrm{AGeV}$. Corresponding mass distributions of $\Sigma$ decays with ToF PID are shown in Fig. 2. Clean topology and accurate mathematics based on the Kalman filter method allow to achieve high signal to background ratios and efficiencies [8].

\section{Summary}

The missing mass method has been realised as an extension of the KF Particle Finder package, which is widely used for searching and reconstructing short-lived particles in several heavy-ion experiments. For the time being 18 decays with neutral daughter have been added to the package.

\section{Acknowledgement}

Work supported by HICforFAIR, FIAS and BMBF.

\section{References}

[1] B. Friman et al., The CBM physics book: Compressed baryonic matter in laboratory experiments, Lect. Notes Phys. 814 (2011)

[2] V. Akishina, Dissertation thesis, Goethe university, Frankfurt am Main (2017)

[3] S. Gorbunov, U. Kebschull, I. Kisel, V. Lindenstruth, and W.F.J. Müller, Computer Physics Communications, 178, No. 5, 374-383 (2008)

[4] I. Kisel, I. Kulakov, and M. Zyzak, IEEE Transactions on Nuclear Science 60, No. 5, 3703 (2013)

[5] M. Zyzak, Dissertation thesis, Goethe university, Frankfurt am Main (2016)

[6] S. A. Bass, et al., Prog. Part. Nucl. Phys. 41, 225-370 (1998)

[7] M. Bleicher, et al., J. Phys. G: Nucl. Part. Phys. 25, 1859-1896 (1999)

[8] V. Akishina, I. Kisel, P. Kisel, P. Senger, I. Vassiliev, and M. Zyzak, XXVI international conference on ultrarelativistic heavy-ion collisions "Quark Matter" (Chicago, USA, February 5-11, 2017) 